\title{
Evaluating Surface Impedance Models for Terahertz Frequencies at Room Temperature
}

\author{
S. Lucyszyn \\ Imperial College London, UK
}

\begin{abstract}
Commercial electromagnetic modeling software employs overly-simplified models for the terahertz simulation of metal structures. For the first time, this paper gives a unique review of various modeling strategies (classical, semiclassical and quantum mechanical based) for normal metals and discusses their limitations with frequency at room temperature.
\end{abstract}

DOI: $10.2529 /$ PIERS061006115842

\section{INTRODUCTION}

High frequency CAD software packages employ overly-simplified models for the electromagnetic simulation of metal structures; using either classical skin-effect or classical relaxation-effect models. At room temperatures, these models are accurate beyond the upper edges of the microwave and sub-millimeter-wave parts of the frequency spectrum, respectively. However, semiclassical models are needed to extend modeling well into the terahertz region or at significantly lower temperatures. Here, issues relating to the specular or diffuse nature of electron reflections at the air-metal interface become apparent at very low temperatures.

Within the near-infrared, visible and ultra-violet parts of the frequency spectrum, Commercial CAD software packages again may employ overly-simplified empirically-fitted relaxation-effect models, which only work over relatively narrow spectral bandwidths. However, to be accurate, an analytical model must be adopted that employs a quantum mechanical treatment, as this takes into account both energy dispersion and electron wavefunctions.

The author has investigated modeling strategies for normal metals. In one study, experimental measurements that suggested the possibility of anomalous room-temperature conduction losses were examined between DC and $12.5 \mathrm{THz}$ [1]. It was found that the classical relaxation-effect model was still valid up to these frequencies. In another study, an elaborate semiclassical model to describe anomalous excess conduction losses at room temperature was found to be completely erroneous [2]. In order to create accurate analytical models, it is important to develop semiclassical modeling strategies [3] or develop quantum mechanical treatments. To this end, and for the first time, this paper will review various approaches to the modeling of normal metals at room temperature. More importantly, their limitations will be discussed in detail. It will be shown that a number of well-know approaches have severe limitations to general applications.

\section{CLASSICAL TREATMENT}

Drude's model of intraband transitions describes an ideal system of free electrons having a spherical Fermi surface. The classical relaxation-effect model takes into account electron-phonon collisions, represented by the following expression for surface impedance, $Z_{S R}$, in terms of Drude's model for intrinsic bulk conductivity, $\sigma_{R}$ :

$$
Z_{S R}=\sqrt{\frac{j \omega \mu_{o} \mu_{r}}{\sigma_{R}+j \omega \varepsilon_{o}}} \quad \text { where } \quad \sigma_{R}=\frac{\sigma_{o}}{(1+j \omega \tau)}
$$

where, angular frequency, $\omega=2 \pi f$; and $f=$ frequency of the driving electric field; $\mu_{o}=$ permeability of free space; $\mu_{r}=$ relative permeability; $\varepsilon_{o}=$ permittivity of free space; $\sigma_{o}=$ intrinsic bulk conductivity at DC; and $\tau=$ phenomenological scattering relaxation time for the free electrons (i.e., mean time between collisions).

Note that the complex operator $+j$ is used throughout and that this replaces $-i$ used in some cited references. Failure to adopt a consistent notation can result in errors. It is found that at room temperature and at sufficiently low frequencies, (1) reduces to the classical skin-effect model:

$$
Z_{S R} \approx \sqrt{\frac{j \omega \mu_{o} \mu_{r}}{\sigma_{0}}}=R_{o}(1+j) \quad \text { when } \quad \omega \tau<<1
$$


where, $R_{o}=$ classical skin-effect surface resistance and the displacement current term can been ignored.

A rigorous investigation into the robustness of the classical relaxation-effect model, when compared to measured data, found that it works well from DC to the lower edge of the mid-infrared frequency range, for normal metals at room temperature [1]. This finding helped to dispel a long-standing myth that an anomalous intrinsic conduction loss in normal metals exists at room temperature.

There are several reasons why the classical relaxation-effect model is not sufficient for a qantitative account of experimental observation, even when interband transitions can be safely ignored or calculated separately:

1) Conduction bands with normal metals can be significantly different from the ideal spherical energy band (that assumes an effective electron mass, $m$ ).

2) Scattering relaxation time is assumed to be independent of energy $E(k)$, where $k=$ wave vector of the electron, even though it depends on both energy and position. For this reason, $\tau$ is considered to be a semi-empirical parameter.

3) A local-response regime is assumed.

For the above reasons, to achieve even better modeling accuracy, it is necessary to move towards the more complicated semiclassical treatment.

\section{SEMICLASSICAL TREATMENT}

It is well known that, at sufficiently high frequencies, the conductivity of normal metals exhibits both temporal (i.e., frequency) and spatial (e.g., one-dimensional) dispersion. Harrison introduced a frequency- and wave number-dependent dielectric function for a semiclassical free-electron gas [4]. This model describes the screened Coulomb potential effect that a spatial charge-density fluctuation has on a free electron as it travels through a periodic lattice of fixed positive ions. It can be helpful to think of induced conduction current within a normal metal as flowing in lamina-type sheets: almost parallel to the surface ( $x$-y-plane) of the conductor and having an amplitude that decays exponentially from its surface into the bulk material. The electric and magnetic fields and conduction current distributions inside the metal have time $t$ and spatial (positive $z$-direction) variations of the form:

$$
e^{(j \omega t-\gamma z)}
$$

where, propagation constant, $\gamma=\alpha+j \beta ; \alpha=$ attenuation constant; $\beta=$ phase constant; and $\gamma=j q$, where modified wave number of the driving electric field, $q=q^{\prime}-j q^{\prime \prime}$.

Wang assumed that the periodic nature of the conduction current density gives rise to a screening potential effect on free electrons as they travel in a direction perpendicular to the surface of the air-metal boundary [5]. With spatial charge-density fluctuations being attributed to lamina-type sheets of conduction currents, as an analogy to a periodic lattice of fixed positive ions, Wang adopted Harrison's screening potential theory. To this end, Harrison's semiclassical expression for intrinsic bulk conductivity, $\sigma_{H}$, was used to calculate Wang's surface impedance, $Z_{S W}[2]$ :

$$
Z_{S W}=\sqrt{\frac{j \omega \mu_{o} \mu_{r}}{\sigma_{H}(s, \omega)}} \text { where } \sigma_{H}(s, \omega)=\sigma_{R}\left\{\frac{-3 j \omega \tau}{s^{2}} \cdot \frac{\left[2 s-\ln \left(\frac{1+s}{1-s}\right)\right]}{2 s j \omega \tau+\left[2 s-\ln \left(\frac{1+s}{1-s}\right)\right]}\right\} \text { and } s=\frac{ \pm j q l_{m}}{(1+j \omega \tau)}
$$

where, $l_{m}=$ mean distance traveled by the electron between collisions (i. e., mean-free path length).

In deriving (4), it was assumed that electrons at all angles, with respect to the metal surface in the half-space, will contribute to the conductivity within this semiclassical free-electron gas analysis [5]. Moreover, the modified wave number considered here is at least two orders of magnitude lower than the Fermi wave number and, therefore, a quantum mechanical analysis is not necessary. It was poorly assumed that with such long wavelengths of potential there is no difference between conductivities for transverse and longitudinal fields in cubic materials [5]. Wang then applied his spatial dispersion theory of excess conduction loss to room temperature measurements of copper, from DC up to $6.7 \mathrm{THz}$, using the measured data at $35 \mathrm{GHz}$ and $70 \mathrm{GHz}$ reported by Tischer $[1,2]$.

It is evident that the expression for intrinsic bulk conductivity, quoted by both Harrison and Wang, is derived for longitudinal wave propagation [2]. As a result, his model has no meaning for surface impedance and excess conduction loss calculations (as they are based on transverse wave 
propagation for normal incidence). Details of a rigorous de-construction of Wang's model have previously been published [2]. From this detailed investigation, it was clearly shown that not only are there serious discrepancies within the general methodology, but even the data on which it is based has unacceptable errors.

An accurate semiclassical treatment was first reported by Reuter and Sondheimer, back in 1948, for transverse wave propagation [6]. Their approach gives an exact solution for all frequencies and temperatures. Moreover, their general methodology can be for any value of specular reflection coefficient, $p$; from diffuse (completely random) scattering, $p=0$, to specular (mirror-type) surface reflections, $p=1$. With the latter, surface impedance $Z s_{R S}$ can be calculated from the following:

$$
\begin{aligned}
& Z s_{R S}=\frac{j \omega \mu_{o} \mu_{r}}{\gamma(o)} \quad \text { where } \gamma(o)=\left[-\left.l_{m} \frac{f(0)}{f^{\prime}(0)}\right|_{p=1}\right]^{-1} \\
& \text { and }\left.\quad \frac{f(0)}{f^{\prime}(0)}\right|_{p=1}=\frac{2}{j \pi(1+j \omega \tau)} \int_{0}^{\frac{j \infty}{(1+j \omega \tau)}} \frac{1}{s^{2}+\frac{\omega^{2} \mu_{o} \mu_{r} \varepsilon_{o} l_{m}^{2}}{(1+j \omega \tau)^{2}}-\xi K(s)} \cdot d s \\
& \text { and } \quad \xi=\frac{j \varsigma}{(1+j \omega \tau)^{3}} ; \quad \varsigma=\frac{3}{4} \omega \mu_{o} \mu_{r} \sigma_{o} l_{m}^{2} ; \quad K(s)=\frac{1}{s^{3}}\left[2 s-\left(1-s^{2}\right) \ln \left(\frac{1+s}{1-s}\right)\right]
\end{aligned}
$$

This modeling approach assumes the following:

1) Skin depth is much less than linear dimensions of metal, thus regarding it as planar and infinite in extent.

2) Normal incidence of propagation, thus simplifying to a one-dimensional problem.

3) Conduction electrons are quasi-free, having a kinetic energy $E(k)=\hbar|k|^{2} / 2 m$ with a parabolic band approximation, where $\hbar=$ modified Planck's constant.

4) Collision mechanism is always described in terms of $l_{m}$.

5) A fraction $p$ of electrons arriving at the surface is scattered specularly, while the rest are scattered diffusely.

6) This one-band free-electron model does not apply to multi-valent metals, in which the electrons occupy more than one energy band (e.g., aluminium or tin).

7) $K(s)$ is derived for transverse conductivity, and holds for the whole $(q, \omega)$ plane, for a spherical energy band.

8) While the semiclassical treatment takes into account partially-filled conduction band energy dispersion, $E(k)$, it ignores electron wavefunctions.

It is interesting to note that when $|s| \ll 1$, e.g., with the long-wavelength limit of $q \rightarrow 0$, then $K(s)=4 / 3$ in $(5)$ and the associated intrinsic bulk conductivity is equal to Drude's model for intrinsic bulk conductivity, $\sigma_{R}$, given in (1).

\section{QUANTUM MECHANICAL TREATMENT}

Zhang and Pan use a quantum mechanical approach to derive, in detail [7], the following expression for the dielectric function of the free-electron gas $[7,8]$ :

$$
\varepsilon_{r}(q, \omega)=1+\frac{2 e^{2}}{q^{2} \varepsilon_{o}} \frac{1}{V} \sum_{k} \frac{f(k)-f(k+q)}{E(k+q)-E(k)-\hbar \omega+j \zeta}
$$

where, $e=$ electron charge; $V=$ volume; $f(k)=$ Fermi-Dirac distribution function. This is a standard derivation and the result is known as the Lindhard dielectric function; also know as the self-consistent-field (SCF) or random phase approximation (RPA) dielectric function [9]. By replacing $f(k+q)$ with $f(-k)$ and taking the long wavelength limit, Zhang and Pan highlight the following $[7,8]$ :

$$
\frac{1}{V} \sum_{k} f(k)=\frac{n}{2} ; \quad \sigma_{o}=\frac{n e^{2} \tau}{m} ; \quad v_{f}=\frac{\hbar k_{f}}{m} ; \quad \zeta=\frac{\hbar}{2 \tau}
$$

where $n=$ electron density, $v_{f}=$ velocity between collisions of the free electron, having kinetic energy at the Fermi level and $k_{f}=$ Fermi wave number; as a means to somehow derive an expression 
for their intrinsic bulk conductivity, in expressions for dielectric function and surface impedance $[7,8]$ :

$$
\begin{aligned}
\varepsilon_{r Z P}(q, \omega) & =1+\frac{\sigma_{Z P}(q, \omega)}{j \omega \varepsilon_{o}} \text { where } \sigma_{Z P}(q, \omega)=\frac{\sigma_{o}}{1+j \omega \tau\left[1-\frac{3}{5}\left(\frac{q l_{m}}{\omega \tau}\right)^{2}\right]} \\
\therefore Z s_{Z P} & =\sqrt{\frac{j \omega \mu_{o} \mu_{r}}{\sigma_{Z P}(q, \omega)+j \omega \varepsilon_{o}}}
\end{aligned}
$$

Unfortunately, the original expression for the dielectric function given in (6) is only valid for longitudinal wave propagation [9]. As a result, the Zhang and Pan model has no meaning for their surface impedance, $Z s_{Z P}$, and excess conduction loss calculations.

It is evident from the publications of Zhang and Pan that they have made the same mistake as Wang, by trying to fit the wrong type of theoretical model to measured data. It has been previously demonstrated that the relaxation-effect model is adequate for characterising the intrinsic frequency dispersive nature of normal metals at room temperature, even into the terahertz frequency range. Indeed, the results from a quantum mechanical model should actually converge onto those from the relaxation-effect model at these frequencies, at room temperature. Since the room temperature results, from the model of Zhang and Pan, deviate from the classical relaxation-effect model at relatively low frequencies then this alone points to a fundamental error.

\section{PROOF OF CONTRADITIONS WITH MODELS (4) AND (8)}

Apart from the lack of any physical insight to justify the need for new models and the obvious misuse of longitudinal wave propagation terms for calculating surface impedance, the work published by Wang and also by Zhang and Pan also share a couple of fundamental contradictions.

\subsection{First Contradiction}

Intrinsic bulk conductivity exhibits spatial dispersion in the non-local response regime and, therefore, has a wave number dependency in reciprocal $q$-space. For one-dimensional propagation along the positive $z$-axis, the general expression for conduction current density, $J c$, in terms of intrinsic bulk conductivity and electric field, $E$, is given by the non-local constitutive equation in real space [10]:

$$
\left.J c_{y}(z)\right|_{p=1}=\int_{-\infty}^{+\infty} \sigma\left(z-z^{\prime}\right) E_{y}\left(z^{\prime}\right) \cdot d z^{\prime}
$$

Now, at the surface of the metal, conduction current density vector, $J c_{y}(0)$, and surface current density vector, $J s$, are related by the propagation constant at the surface, $\gamma(0)$, as follows:

$$
J c_{y}(0)=\gamma(0) J s \quad \text { where } \quad J s=n \times H_{x}(0)
$$

where $n=$ unit vector pointing out normal to the surface of the metal and $H_{x}(0)=$ magnetic field vector at the surface of the metal. Equation (9) can be expressed in terms of a simple convolution (denoted by the symbol ${ }^{*}$ ) of the intrinsic bulk conductivity and electric field as follows:

$$
\left.\therefore J c_{y}(z)\right|_{p=1} \equiv\left\{\sigma(z)^{*} E_{y}(z)\right\}
$$

But, surface impedance for normal incidence is related to the electric and magnetic fields as follows using Ohms law $[9,10]$ :

$$
Z s=\frac{E_{y}(0)}{H_{x}(0)}
$$

It can be easily seen that determining surface impedance is not so straight forward in the non-local response regime, since:

$$
\left.\left\{\sigma(z)^{*} E_{y}(z)\right\}\right|_{z=0} \equiv \gamma(0) H_{x}(0)
$$

Now, taking the Fourier transform of both sides of (11) gives the non-local constitutive equation in reciprocal $q$-space [10]:

$$
\left.J c(q)\right|_{p=1}=\sigma(q) E(q)
$$


Surface impedance, by its very nature, must be expressed within real space and so (14) is of no direct use. In the local response regime, however, the generalised conductivity behaves like a $\delta$-function, i.e., $\sigma\left(z-z^{\prime}\right) \rightarrow \sigma \delta\left(z-z^{\prime}\right)$.

$$
\therefore \int_{-\infty}^{+\infty} \delta\left(z-z^{\prime}\right) E_{y}\left(z^{\prime}\right) \cdot d z^{\prime}=E_{y}(z) \quad \text { and }\left.\quad J c_{y}(z)\right|_{p=1} \rightarrow \sigma E_{y}(z)
$$

Therefore, in the local response regime, which represents the long-wavelength limit $q \rightarrow 0$ in reciprocal $q$-space, and ignoring displacement current, the simple expression for surface impedance can be easily determined using (10), (12) and (15):

$$
Z s=\frac{\gamma(0)}{\sigma}=\sqrt{\frac{j \omega \mu_{o} \mu_{r}}{\sigma}} \quad \text { where } \quad \sigma \neq f(q)
$$

Evidently, (16) could only have been derived here if the intrinsic bulk conductivity is in the local response regime. Since Wang's semiclassical model and the work by Zhang and Pan both adopt (16) with intrinsic bulk conductivity having spatial dispersion, i.e., in a non-local response regime, this is direct proof of a fundamental contradiction.

\subsection{Second Contradiction}

Angular frequency $\omega$ and modified wave number $q$ are assumed to be real variables by both Wang [5] and also by Zhang [11], but this must be a complex variable when modeling normal metals at room temperatures and at frequencies below the plasma frequency, as previously stated by Lucyszyn [2]. In general, either the angular frequency $\omega$ or the modified wave number $q$ must be a complex term, in order to account for an exponentially decaying wave as it propagates into the metal. The dispersion relations for electromagnetic waves in an isotropic homogeneous medium has either $q$ being complex and $q=f(\omega)$, where $\omega$ is real, or $\omega$ being complex and $\omega=f(q)$, where $q$ is real [9]. Only if there is no dissipation of electromagnetic energy can both $q$ and $\omega$ be real [9]. Therefore, since both are treated as real variables, this contradicts the principle of conservation of energy for wave propagation within a normal metal; once again challenging the validity of (4) and (8).

\section{CONCLUSIONS}

It would be inappropriate to use either Wang's semiclassical model or the work by Zhang and Pan to support the view that anomalous intrinsic frequency dispersion exists within normal metals at room temperature. When the findings from this investigation are combined with those recently published on the lack of experimental evidence, any myth associated with anomalous behavior at room temperature can be finally dispelled; the relaxation-effect model is sufficiently accurate to describe the natural behavior of normal metals at room temperature. It has already been shown that measured data up to $12.5 \mathrm{THz}$ actually fits the classical relaxation-effect model quite well [1]. This is good news for those working between circa $30 \mathrm{GHz}$ and $12 \mathrm{THz}$. For example, within future measurement systems, the mathematically simple Drude model can be used to characterize the surface impedance for calibration standards (with accurate values for only $\sigma_{o}$ and $\tau$ being needed by the metrologist). This approach would lead to much more accurately calibrated sub-mm-wave measurement systems. For even greater accuracy, the complicated semiclassical approach based on the work of Reuter and Sondheimer are recommended, especially when modeling is to be undertaken for applications well below room temperature.

\section{REFERENCES}

1. Lucyszyn, S., "Investigation of anomalous room temperature conduction losses in normal metals at terahertz frequencies," IEE Proc. - Microwaves, Antennas and Propagation, Vol. 151, No. 4, 321-329, Aug. 2004.

2. Lucyszyn, S., "Investigation of Wang's model for room temperature conduction losses in normal metals at terahertz frequencies," IEEE Transactions on Microwave Theory Tech., Vol. 53, No. 4, 1398-1403, Apr. 2005.

3. Lucyszyn, S., "Accurate CAD modelling of metal conduction losses at terahertz frequencies," 11th IEEE International Symposium on Electron Devices for Microwave and Optoelectronic Applications (EDMO2003), 180-185, Orlando, USA, Nov. 2003.

4. Harrison, W. A., Solid State Theory, 280-290, McGraw-Hill, 1970. 
5. Wang, Y.-C., "The screening potential theory of excess conduction loss at millimeter and submillimeter wavelengths," IEEE Trans. on Microwave Theory Tech., Vol. MTT-26, No. 11, 858-861, Nov. 1978.

6. Reuter, G. E. H. and E. H. Sondheimer, "The theory of the anomalous skin effect in metals," Proc. Royal Society, Vol. A195, 336-364, London, 1948.

7. Zhang, X., W. Pan, and S. Lucyszyn, "A new theoretical modeling of surface resistance in normal metals at terahertz frequencies," International Journal of Infrared and Millimeter Waves, Vol. 25, No. 11, 1611-1620, Springer Science+Business Media Inc., Nov. 2004.

8. Pan, W. and X. Zhang, "Study of excess conduction loss in normal metals at/below submillimeter wavelengths," International Journal of Infrared and Millimeter Waves, Vol. 27, No. 3, 455-464, Springer Science+Business Media Inc., Mar. 2006.

9. Fuchs, R. and P. Halevi, "Basic concepts and formalism of spatial dispersion," Spatial Dispersion in Solids and Plasmas, Halevi, P. (Editor), Chapter 1, Published by North-Holland, 1992.

10. Grosso, G. and G. P. Parravicini, Solid State Physics, Chapter 11, 389-414, Published by Academic Press, 2000.

11. Zhang, X., Private Communication, Sep. 2004. 